\title{
Image Processing for Diffusion Tensor Magnetic Resonance Imaging
}

\author{
C.-F. Westin, S. E. Maier, B. Khidhir, P. Everett, F. A. Jolesz, and R. Kikinis \\ Surgical Planning Lab, Radiology \\ Brigham and Women's Hospital, Harvard Medical School, Boston \\ westin@bwh.harvard.edu
}

\begin{abstract}
This paper describes image processing techniques for Diffusion Tensor Magnetic Resonance. In Diffusion Tensor MRI, a tensor describing local water diffusion is acquired for each voxel. The geometric nature of the diffusion tensors can quantitatively characterize the local structure in tissues such as bone, muscles, and white matter of the brain. The close relationship between local image structure and apparent diffusion makes this image modality very interesting for medical image analysis.

We present a decomposition of the diffusion tensor based on its symmetry properties resulting in useful measures describing the geometry of the diffusion ellipsoid. A simple anisotropy measure follows naturally from this analysis. We describe how the geometry, or shape, of the tensor can be visualized using a coloring scheme based on the derived shape measures. We show how filtering of the tensor data of a human brain can provide a description of macrostructural diffusion which can be used for measures of fiber-tract organization. We also describe how tracking of white matter tracts can be implemented using the introduced methods. These methods offers unique tools for the in vivo demonstration of neural connectivity in healthy and diseased brain tissue.
\end{abstract}

\section{Introduction}

Diffusion Tensor Magnetic Resonance Imaging (DT-MRI) is a recent MR imaging modality. In Diffusion Tensor MRI, a tensor describing local water diffusion is acquired for each voxel. Diffusion in tissue can be anisotropic depending on the characteristics of the tissue. For example in the white matter fiber tracts the diffusion is mainly in the direction of the fibers. In areas with fluid, such in the CSF filled ventricles, the diffusion is spherical, i.e. isotropic. The advent of robust diffusion tensor imaging techniques has prompted the development of quantitative measures for describing the diffusion anisotropy. A good review by Basser and Pierpaoli can be found in [1].

Since MRI methods in general always obtain a macroscopic measure of a microscopic quantity which necessarily entails intravoxel averaging, the voxel dimensions influence the measured diffusion tensor at any particular location in the brain. 
Factors which would affect the shape of the apparent diffusion tensor (shape of the diffusion ellipsoid) in the white matter include the density of fibers, the degree of myelination, the average fiber diameter and the directional similarity of the fibers in the voxel. The geometric nature of the measured diffusion tensor within a voxel is thus a meaningful measure of fiber tract organization.

With current conventional proton magnetic resonance imaging (MRI) techniques, the white matter of the brain appears to be a remarkably homogeneous tissue without any suggestion of the complex arrangement of fiber tracts. Although the individual axons and the surrounding myelin sheaths cannot be revealed with the limited spatial resolution of in vivo imaging, distinct bands of white matter fibers with parallel orientation may be distinguished from others running in different directions if MRI techniques are sensitized to water diffusion and the preferred direction of diffusion is determined.

Water diffusion in tissue due to Brownian motion is random but some structural characteristics of tissues may limit diffusion. In the white matter, the mobility of the water is restricted in the directions perpendicular to the axons which are oriented along the fiber tracts. This anisotropic diffusion is due to the presence of tightly packed multiple myelin membranes encompassing the axon. Myelination is not essential for diffusion anisotropy of nerves as shown in studies of nonmyelinated garfish olfactory nerves [3] and anisotropy exists in brains of neonates before the histological appearance of myelin [16] but myelin is widely assumed to be the major barrier to diffusion in myelinated fiber tracts. Therefore the demonstration of anisotropic diffusion in brain by magnetic resonance has opened the way to explore noninvasively the structural anatomy of the white matter in vivo $[8,4,1,10]$.

\section{Materials and Methods}

In this work we applied a modified version of the recently proposed Line Scan Diffusion Imaging (LSDI) technique [7]. This method, like the commonly used diffusion-sensitized, ultrafast, echo-planar imaging (EPI) technique [12] is relatively insensitive to bulk motion and physiologic pulsations of vascular origin. But unlike EPI, LSDI exhibits minimal image distortion, does not require cardiac gating, head restraints or post-processing image correction, and can be implemented without specialized hardware on all standard MRI scanners.

Here, we present a quantitative characterization of the geometric nature of the diffusion tensors, a method for characterization of marcostructural diffusion properties, and a display method for showing clear and detailed in vivo images of human white matter tracts. The orientation and distribution of most of the known major fiber tracts can be identified using these methods.

\subsection{Imaging Parameters}

Our data were acquired at the Brigham and Women's Hospital on a GE Signa 1.5 Tesla Horizon Echospeed 5.6 system with standard 2.2 Gauss/cm field gradients. 
The time required for acquisition of the diffusion tensor data for one slice was 1 min; no averaging was performed. Imaging parameters were: effective $\mathrm{TR}=2.4 \mathrm{~s}$, $\mathrm{TE}=65 \mathrm{~ms}, b_{\text {high }}=750 \mathrm{~s} / \mathrm{mm}^{2}, b_{\text {low }}=5 \mathrm{~s} / \mathrm{mm}^{2}$, field of view $22 \mathrm{~cm}$, effective voxel size $4.8 \times 1.6 \times 1.5 \mathrm{~mm}^{3}, 6 \mathrm{kHz}$ readout bandwidth, acquisition matrix $128 \times 128$.

The gradient cycle in the LSDI interleaving scheme was modified to provide acquisition of more gradient directions and to allow elimination of the crusher gradients. Instead of alternating merely between high and low gradient strengths, the modified sequence cycled through eight configurations of the diffusion gradients. In all other respects it was identical to the sequence described in [7].

\subsection{Calculation of Tensors}

For each slice, eight images are collected with different diffusion weightings and noncollinear gradient directions. If $S_{0}$ represents the signal intensity in the absence of a diffusion-sensitizing field gradient and $S$ the signal intensity in the presence of gradient $\mathbf{g}=\left(g_{x}, g_{y}, g_{z}\right)^{T}$, the equation for the loss in signal intensity due to diffusion is given by the Stejskal-Tanner formula:

$$
\ln (S)=\ln \left(S_{0}\right)-\gamma^{2} \delta^{2}(\Delta-\delta / 3) \mathbf{g}^{T} \mathbf{D g},
$$

where $\gamma$ is the gyromagnetic ratio of hydrogen ${ }^{1} \mathrm{H}$ (protons), $\delta$ is the duration of the diffusion sensitizing gradient pulses and $\Delta$ is the time between the centers of the two gradient pulses. The eight images provide eight equations for $S$ in each voxel which are solved in a least-squares sense for the $6+1$ unknowns: the six independent components of the symmetric diffusion tensor, $\mathbf{D}$, and $S_{0}$. In the LSDI sequence, it is easy to show that cross terms between the slice select gradient for the $180^{\circ}$ pulse and the diffusion sensitizing gradients account for less than $0.1 \%$ of the diffusion weighting, and have therefore been neglected here. Diffusion attenuation due to imaging gradients is already factored into $S_{0}$, as is $\mathrm{T}_{2}$ weighting.

\subsection{Geometrical Measures of Diffusion}

In order to relate the measure of diffusion anisotropy to the structural geometry of the tissue a mathematical description of diffusion tensors and their quantification is necessary [1]. First, a complete diffusion tensor, D, is calculated (Equation 1) for each voxel. Using the symmetry properties of the diffusion ellipsoid we decomposed the diffusion tensor, and from the tensor basis assigned scalar measures, describing the linearity and the anisotropy, to each voxel [15].

The diffusion tensor can be visualized using an ellipsoid where the principal axes correspond to the directions of the eigenvector system. Let $\lambda_{1} \geq \lambda_{2} \geq \lambda_{3} \geq 0$ be the eigenvalues of the symmetric tensor $\mathbf{D}$, and let $\hat{\mathbf{e}}_{i}$ be the normalized eigenvector corresponding to $\lambda_{i}$.

$$
\mathbf{D}=\lambda_{1} \hat{\mathbf{e}}_{1} \hat{\mathbf{e}}_{1}^{T}+\lambda_{2} \hat{\mathbf{e}}_{2} \hat{\mathbf{e}}_{2}^{T}+\lambda_{3} \hat{\mathbf{e}}_{3} \hat{\mathbf{e}}_{3}^{T}
$$


Diffusion can be divided into three basic cases depending on the rank, of the representation tensor:

1) Linear case $\left(\lambda_{1} \gg \lambda_{2} \simeq \lambda_{3}\right)$ : diffusion is mainly in the direction corresponding to the largest eigenvalue,

$$
\mathbf{D} \simeq \lambda_{1} \mathbf{D}_{l}=\lambda_{1} \hat{\mathbf{e}}_{1} \hat{\mathbf{e}}_{1}^{T}
$$

2) Planar case $\left(\lambda_{1} \simeq \lambda_{2} \gg \lambda_{3}\right)$ : diffusion is restricted to a plane spanned by the two eigenvectors corresponding to the two largest eigenvalues,

$$
\mathbf{D} \simeq 2 \lambda_{1} \mathbf{D}_{p}=\lambda_{1}\left(\hat{\mathbf{e}}_{1} \hat{\mathbf{e}}_{1}^{T}+\hat{\mathbf{e}}_{2} \hat{\mathbf{e}}_{2}^{T}\right) .
$$

3) Spherical case $\left(\lambda_{1} \simeq \lambda_{2} \simeq \lambda_{3}\right)$ : isotropic diffusion,

$$
\mathbf{D} \simeq 3 \lambda_{1} \mathbf{D}_{s}=\lambda_{1}\left(\hat{\mathbf{e}}_{1} \hat{\mathbf{e}}_{1}^{T}+\hat{\mathbf{e}}_{2} \hat{\mathbf{e}}_{2}^{T}+\hat{\mathbf{e}}_{3} \hat{\mathbf{e}}_{3}^{T}\right) .
$$

In general, the diffusion tensor $\mathbf{D}$ will be a combination of these cases. Expanding the diffusion tensor using these cases as a basis gives:

$$
\begin{aligned}
\mathbf{D}= & \lambda_{1} \hat{\mathbf{e}}_{1} \hat{\mathbf{e}}_{1}^{T}+\lambda_{2} \hat{\mathbf{e}}_{2} \hat{\mathbf{e}}_{2}^{T}+\lambda_{3} \hat{\mathbf{e}}_{3} \hat{\mathbf{e}}_{3}^{T} \\
= & \left(\lambda_{1}-\lambda_{2}\right) \hat{\mathbf{e}}_{1} \hat{\mathbf{e}}_{1}^{T}+\left(\lambda_{2}-\lambda_{3}\right)\left(\hat{\mathbf{e}}_{1} \hat{\mathbf{e}}_{1}^{T}+\hat{\mathbf{e}}_{2} \hat{\mathbf{e}}_{2}^{T}\right) \\
& +\lambda_{3}\left(\hat{\mathbf{e}}_{1} \hat{\mathbf{e}}_{1}^{T}+\hat{\mathbf{e}}_{2} \hat{\mathbf{e}}_{2}^{T}+\hat{\mathbf{e}}_{3} \hat{\mathbf{e}}_{3}^{T}\right) \\
= & \left(\lambda_{1}-\lambda_{2}\right) \mathbf{D}_{l}+\left(\lambda_{2}-\lambda_{3}\right) \mathbf{D}_{p}+\lambda_{3} \mathbf{D}_{s}
\end{aligned}
$$

where $\left(\lambda_{1}-\lambda_{2}\right),\left(\lambda_{2}-\lambda_{3}\right)$ and $\lambda_{3}$ are the coordinates of $\mathbf{D}$ in the tensor basis $\left\{\mathbf{D}_{l}, \mathbf{D}_{p}, \mathbf{D}_{s}\right\}$. A similar tensor shape analysis has proven to be useful in a number of computer vision applications $[13,14,6]$.

As described, the relationships between the eigenvalues of the diffusion tensor can be used for classification of the diffusion tensor according to geometrically meaningful criteria. By using the coordinates of the tensor in our new basis measures are obtained of how close the diffusion tensor is to the generic cases of line, plane and sphere. The generic shape of a tensor is obtained by normalizing with a magnitude measure of the diffusion. Here we define this magnitude as the largest eigenvalues of the tensor. This gives for the linear, planar and spherical measures:

$$
\begin{gathered}
c_{l}=\frac{\lambda_{1}-\lambda_{2}}{\lambda_{1}} \\
c_{p}=\frac{\lambda_{2}-\lambda_{3}}{\lambda_{1}} \\
c_{s}=\frac{\lambda_{3}}{\lambda_{1}} \\
c_{l}+c_{p}+c_{s}=1
\end{gathered}
$$

An anisotropy measure describing the deviation from the spherical case is achieved as follows:

$$
c_{a}=c_{l}+c_{p}=1-c_{s}=1-\frac{\lambda_{3}}{\lambda_{1}}
$$




\subsection{Relaxation of Data}

For many medical image analysis procedures preprocessing of the data in order to reduce the image noise level is important [5]. For diffusion data, regularization based on a Markov model was used to achieve more stable directionality information for tracking white matter fiber tracts [11].

In this paper we argue for staying in the tensor domain when processing simplifies cleaning up of the data. Simple averaging of the tensor components has proven to be a robust relaxation method. One reason for the usefulness of this approach is that tensors have more degrees of freedom compared to scalars and vectors. This is illustrated in Figure 1. Adding two vectors (a) and (b) results in a new vector (c). The data (a) and (b) and the result (c) are of the same order. However, adding two rank 1 tensors (d) and (e), e.g. diffusion tensors from two differently oriented white matter tracts, results in a rank 2 tensor (f), i.e. the output has more degrees of freedom than the input tensors and describes the plane in which diffusion is present. Averaging of tensors are different from averaging a vector field:

- The average of a set of vectors gives the "mean event"

- The average of a set of tensors gives the "mean event" and the "range of the present events"

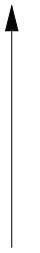

(a)

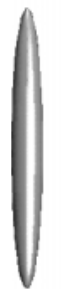

(d) (b)

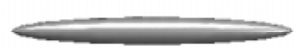

(e)

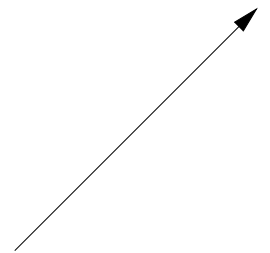

(c)

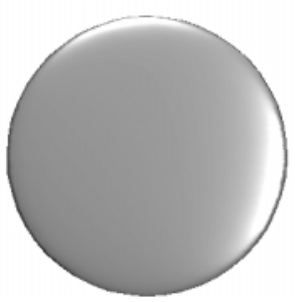

(f)

Fig. 1. Vector and tensor summation. Two vectors, (a) and (b), and their sum (c). Two diffusion tensors, (d) and (e), of rank close to 1 visualized as ellipsoids with eigenvectors forming principal axes. The summation of the two tensors gives a rank 2 tensor (f). 
Figure 2 shows two stylized 2D examples illustrating the effect of relaxation of a diffusion tensor field. (a) Relaxation of a field that contains inconsistent data (left) gives a result of almost round ellipses (right). In (b) relaxation of a field containing data with a clear bias in one direction resulting in a more stable estimate of the directionality of the field.

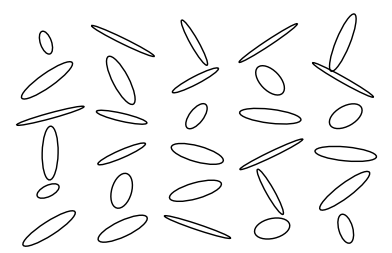

(a)
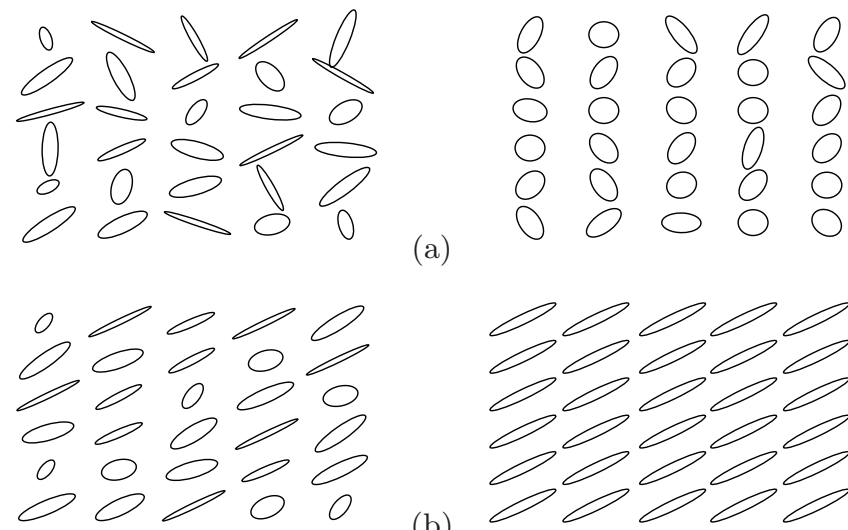

(b)

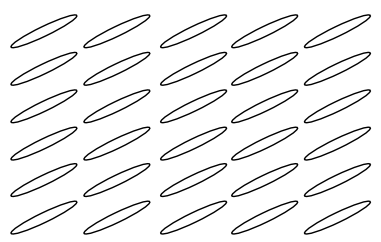

Fig. 2. Stylized 2D examples illustrating the effect of relaxation of a diffusion tensor field. (a) Relaxation of a field that contains inconsistent data (left) gives a result of almost round ellipses (right). (b) Relaxation of a field containing data with a clear bias in one direction.

\subsection{Macrostructural Diffusive Similarities}

In the previous section we characterized diffusion isotropy and anisotropy within a voxel. Here, we will introduce a new method for examining the pattern or distribution of diffusion within an image volume.

Basser and Pierpaoli proposed a scalar measure for macrostructural diffusive similarity based on summing tensor inner products between the center voxel tensor and its neighbors [2]. As in a vector case, the inner product between two tensors measures their degree of similarity.

We will use this idea, and show that averaging the diffusion tensor field has a close relation to this approach. A weighted linear sum of tensor inner products operation over an image volume can be expressed as

$$
\sum_{k}\left\langle\mathbf{D}(\mathbf{x}), a\left(\mathbf{x}_{k}\right) \mathbf{D}\left(\mathbf{x}_{k}\right)\right\rangle=\left\langle\mathbf{D}(\mathbf{x}), \sum_{k} a\left(\mathbf{x}_{k}\right) \mathbf{D}\left(\mathbf{x}_{k}\right)\right\rangle=\left\langle\mathbf{D}(\mathbf{x}), \mathbf{D}_{a}(\mathbf{x})\right\rangle
$$

where the brackets denote inner product, $a\left(\mathbf{x}_{k}\right)$ is a spatial mask defining the local image volume of interest around $\mathbf{x}, k$ is an index ordering the voxel in this volume. $\mathbf{D}_{a}$ is a weighted average of the tensors in the neighborhood under the mask $a, \sum_{k} a\left(\mathbf{x}_{k}\right) \mathbf{D}\left(\mathbf{x}_{k}\right)$. Or, in other words, the filtered output from applying 


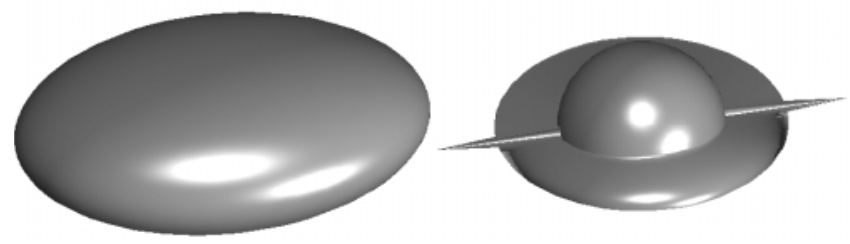

Fig. 3. Comparison of an ellipsoid and a composite shape depicting the same tensor with eigenvalues $\lambda_{1}=1, \lambda_{2}=0.7$, and $\lambda_{3}=0.4$.

the filter $a\left(\mathbf{x}_{k}\right)$ to the data $\mathbf{D}\left(\mathbf{x}_{k}\right)$. Note that averaging of a diffusion tensor field and then deriving a scalar measure from the averaged field is not the same as averaging a scalar field derived from the original field.

Equation 11 shows that the scalar macrostructural measure is an inner product between a diffusion tensor with a blurred version of itself. This can be seen as inner products between tensors in a scale pyramid where the tensors with highest spatial resolution, and tensors at a level in the scale pyramid corresponding to the size of interest (scale obtained by blurring with mask $a$ ). Figure 4 (rightmost column) shows three level of such a pyramid.

The rank of the average tensor $\mathbf{D}_{a}$ describes the complexity of the macroscopic diffusion structure. If the rank is close to one, the structure is highly linear which will be the case in regions of bundles of fibers having the same direction. If the rank is two, fibers are crossing in a plane, or the underlying diffusitivity is planar. Instead of carrying out the inner product in Equation 11, to get a scalar value for similarity, we use the tensor $\mathbf{D}_{a}$ as a description of macrostructural diffusion.

\subsection{Visualization of Diffusion Tensors}

A 3D diffusion tensor can be visualized using an ellipsoid where the principal axes correspond to the tensor's eigenvector system. However, it is difficult to distinguish between an edge-on, flat ellipsoid and an oblong one using the surface shading information. Similar ambiguity exists between a face-on, flat ellipsoid and a sphere. We propose two techniques for the visualization of tensor fields that overcome the problems with ellipsoids. Figure 3 compares the ellipsoidal representation of a tensor with a composite shape whose linear, planar, and spherical components are scaled according to $c_{l}, c_{p}$, and $c_{s}$.

Additionally, coloring based on the shape measures $c_{l}, c_{p}$, and $c_{s}$ can be used for visualization of shape. Figure 6 shows a coloring scheme where the color is interpolated between the blue linear case, the yellow planar case, and the red spherical case. 


\subsection{Eigenvalue Transformations}

Smoothing a diffusion tensor field removes high frequency noise and as discussed above stabilizes the field. In this section we discuss methods that changes the eigenvalues of the tensors in a way that further can stabilize the data. Let $\mathbf{D}=\sum_{k=1}^{3} \lambda_{k} \hat{\mathbf{e}}_{k} \hat{\mathbf{e}}_{k}^{T}$. The first method is based on direct manipulation of the eigenvalues $\lambda_{k}$.

$$
\mathbf{T}=\sum_{k=1}^{3} f_{k}\left(\lambda_{k}\right) \hat{\mathbf{e}}_{k} \hat{\mathbf{e}}_{k}^{T}
$$

where $f_{k}$ are scalar functions. The function $f$ can for example be a thresholding operator which results in decreased rank of the tensors when the eigenvalues are smaller than the specified threshold. Another method is a "max shape" operator,

$$
\mathbf{T}=\left\{\begin{array}{lll}
c_{l} \mathbf{D}_{l} & \text { if } & c_{l}>c_{p}, c_{s} \\
c_{p} \mathbf{D}_{p} & & c_{p}>c_{l}, c_{s} \\
c_{s} \mathbf{D}_{s} & & c_{s}>c_{l}, c_{p}
\end{array}\right.
$$

This operation forces the tensor into the closest of the three generic shapes line, plane, and sphere.

\subsection{Tracking White Matter Tracts}

This section gives a sketch of a tracking algorithm that uses the diffusion tensors as operators. Let $\mathbf{x}_{0}$ be the initial seed point, and $\mathbf{v}_{0}$ be the seed direction, e.g. the eigenvector corresponding to the largest eigenvalue. A tracking sequence $\left\{\mathbf{x}_{0}, \mathbf{x}_{1}, \ldots\right\}$ can then be obtained by the following iteration formula:

$$
\begin{aligned}
& \mathbf{x}_{k+1}=\mathbf{x}_{k}+\alpha \hat{\mathbf{v}}_{k} \\
& \mathbf{v}_{k+1}=\mathbf{T}\left(\mathbf{x}_{k+1}\right) \mathbf{v}_{k}
\end{aligned}
$$

where $\mathbf{T}\left(\mathbf{x}_{k+1}\right)$ is the tensor in spatial position $\mathbf{x}_{k+1}$. The idea here is based on that $\mathbf{v}_{k}$ will turn towards the largest eigenvector of the tensor $\mathbf{T}\left(\mathbf{x}_{k+1}\right)$. When the tensor has lower than full rank, it will act as a projection operator. When rank one, the vector $\mathbf{v}_{k}$ will be projected onto $\left(\mathbf{e}_{1}\right)_{k+1}$. Further, when the tensor is spherical, $\mathbf{v}_{k}$ will not turn since all vectors are eigenvectors.

\section{Results}

When applied to white matter, the linear measure, $c_{l}$, reflects the uniformity of tract direction within a voxel because it will be high only if the diffusion is restricted in two orthogonal directions. The anisotropy measure, $c_{a}$, indicates the relative restriction of the diffusion in the most restricted direction and will emphasize white matter tracts which within a voxel exhibit at least one direction of relatively restricted diffusion. Figure 4 shows the three geometrical measures and the anisotropy index. Major white matter tracts such as the corpus callosum 
show high linearity. In Figure 5 a diffusion tensor field from an axial slice of the brain is shown (left) and the averaged tensor field (right). The window, $a$, used was a $5 \times 5 \times 3$ Gaussian window with standard deviation equal to 1 , defined in the in-plane resolution of the image. Since the out-of-plane resolution is slightly less than half the in-plane resolution, there is almost no smoothing performed between the slices. The original field (left) and the averaged field (right) have been weighted with their linear diffusion measure, $c_{l}$ (Equation 8), respectively. This procedure can be used to measure fiber tract organization quantitatively. The size of the filter should be chosen in relation to the size of the fiber tracts of interest.

Figure 7 shows the effect of the two eigenvalue operations described in section 2.7. The left image shows the input data, a simulated tensor field of crossing white matter tracts. Due to partial voluming effects, the tensors in the area where the fibers are crossing have planar shape. The middle image shows the effect of smoothing the field with a $5 \times 5$ Gaussian kernel followed by a remapping the eigenvalues when $f$ is a thresholding operator (Equation 12). The threshold was set to $20 \%$ of the largest eigenvalue, $0.2 \lambda_{1}$. The right image shows the effect of the max shape operator on the same input data.

Figure 8 shows the result of tracking three crossing fiber tracts. A $5 \times 5 \times 5$ Gaussian operator followed by the max shape operator was used to stabilize the data. Each trace line is a composition of the results from both seed directions $\mathbf{e}_{1}$ and $-\mathbf{e}_{1}$ joined together.

\section{Conclusions}

We have proposed measures classifying diffusion tensors into three generic cases based on a tensor basis expansion. When applied to white matter the linear index shows uniformity of tract direction within a voxel while the anisotropic index quantifies the deviation from spatial homogeneity. The non-orthogonal tensor basis chosen is intuitively appealing since it is based on three simple, yet descriptive, geometrically meaningful cases.

We have described how tensor diffusion data can be processed without reverting to the use of only scalar measures of the tensor data. By staying in the tensor domain, cleaning up of the data can be done meaningfully with simple methods such as smoothing. We discuss addition of tensors geometrically and argue that adding tensors and vectors are different in that tensor summation gives more than the "mean" event due to more degrees of freedom. By using the geometric diffusion measures on locally averaged tensors local directionality consistency can be determined (e.g. existence of larger fiber tracts). We have proposed that this averaging approach can be used to derive a tensor field that can be used to describe macrostructural features in the tensor diffusion data. The linear measure $c_{l}$ derived from the averaged tensor field can for example be used for quantitative evaluation of fiber tract organization. We also have described how non-linear operations can be used to remap the eigenvalues of the diffusion tensors and given a sketch of how this can be used for tracking white matter tracts. 
linear
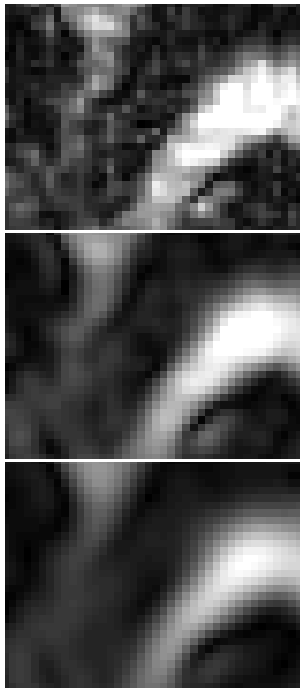

planar
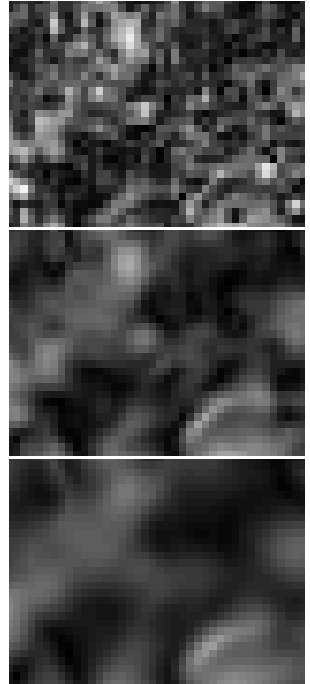

spherical

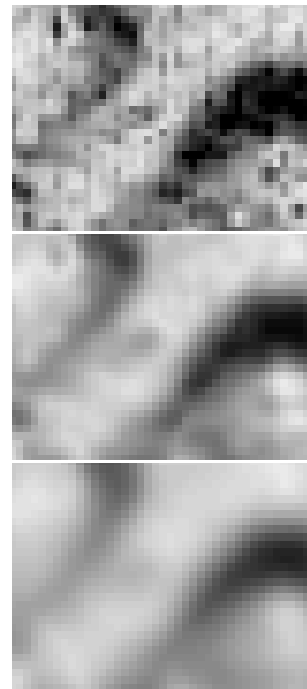

tensor map

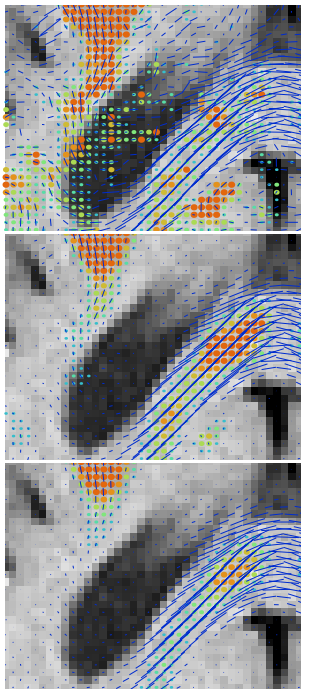

Fig. 4. Axial brain images showing the three geometrical measures and diffusion tensor maps with three different smoothing parameters. Top: shows the geometrical measures and the tensor map derived from the original data. Middle: shows the same measures derived from data that has been averaged with 9x9x3 Gaussian kernel. Bottom: from data averaged with a 15x15x5 Gaussian kernel. The rightmost column shows the tensors. The blue headless arrows represent the in-plane components of $c_{l} \hat{\mathbf{e}}_{1}$. The out-of-plane components of $c_{l} \hat{\mathbf{e}}_{1}$ are shown in colors ranging from green through yellow to red, with red indicating the highest value for this component. Display technique from [9]

\section{Acknowledgements}

This work was funded in part by the Wenner-Gren Foundation, Sweden, the Whitaker Foundation, NIH grants P41-RR13218-01, R01-RR11747-01A, P01CA67165-03. 

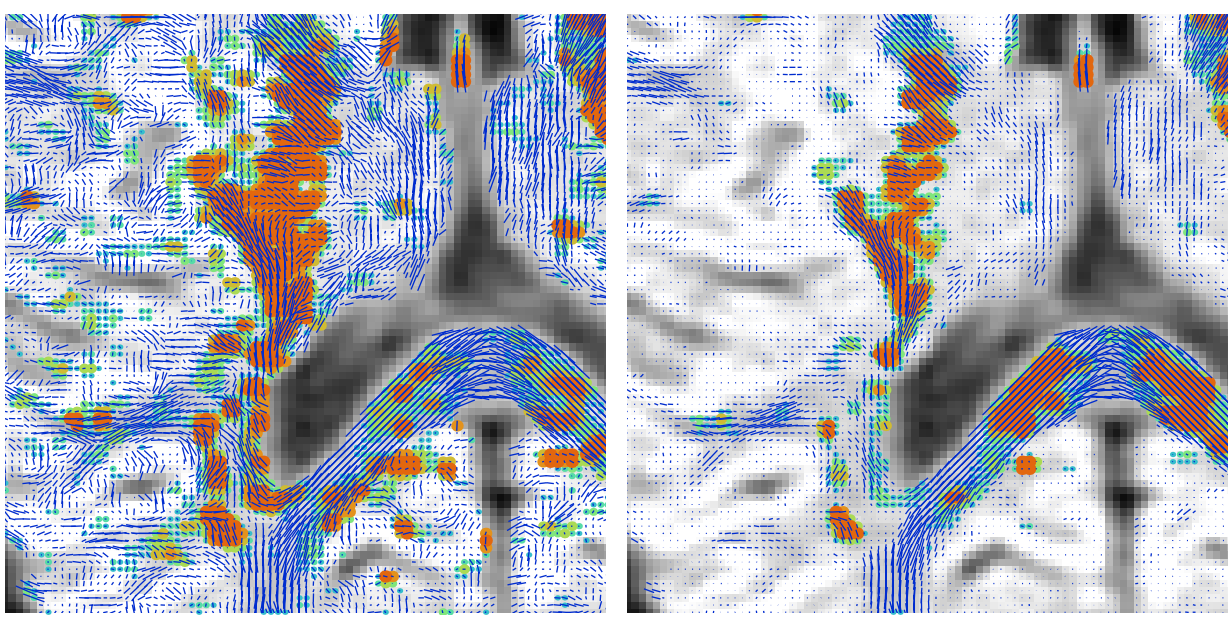

Fig. 5. Left: Diffusion tensors, weighted with their linear measure $c_{l}$, from an axial slice of a human brain. Right: Averaged diffusion tensors using a $5 \times 5 \times 3$ Gaussian kernel weighted with their linear measure $c_{l}$.
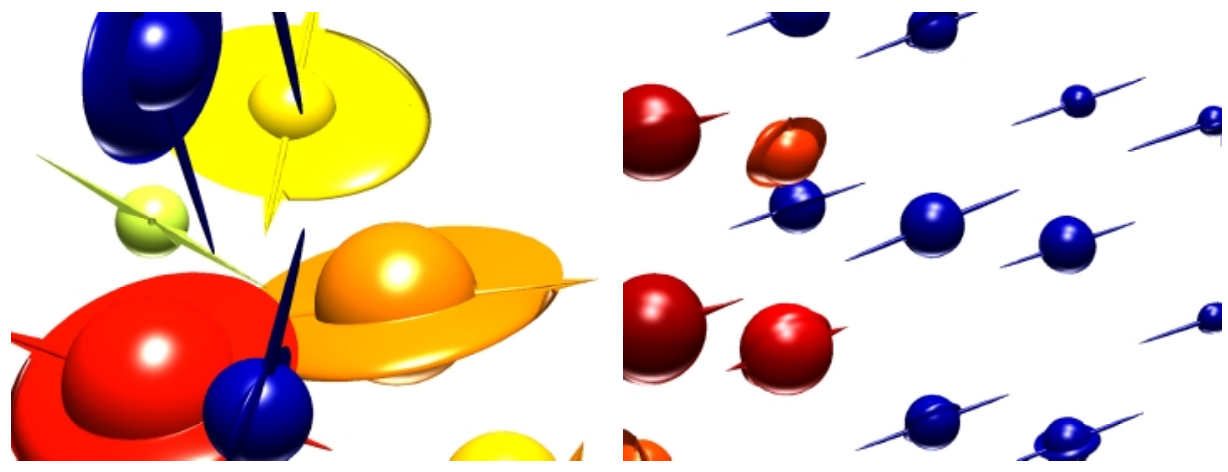

Fig. 6. Visualization of diffusion tensors. The tensors are color coded according to the shape: linear case is blue, planar case is yellow, and spherical case is red. The radius of the sphere is the smallest eigenvalue of the diffusion tensor, the radius of the disk is second largest and the length of the rod is twice the largest eigenvalue. 


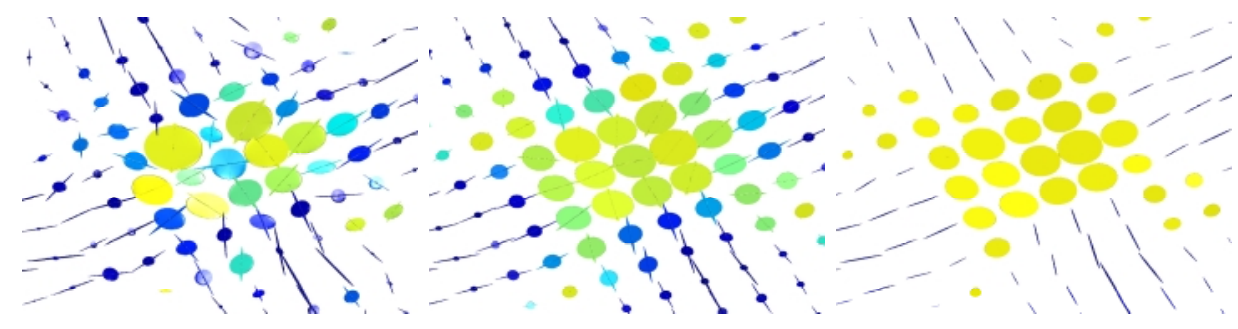

Fig. 7. Left: Simulated diffusion tensor data of two crossing white matter tracts. Middle The effect of thresholding the eigenvalues at $20 \%$ of $\lambda_{1}$. Right: The effect of the max shape operator.
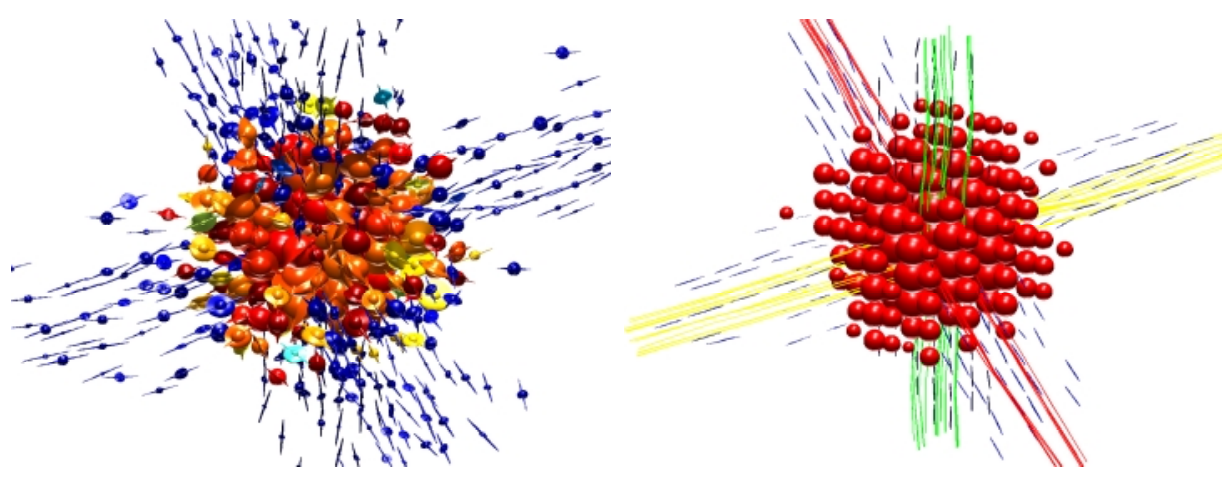

Fig. 8. Left: Original tensor data. Right: Tracking fibers after applying the max shape operator. 10 points were randomly seeded at one end of each of the three branches. Note how the stream lines "tunnel" trough the area in the center where the information of directionality is uncertain. 


\section{References}

1. P.J. Basser. Inferring microstructural features and the physiological state of tissues from diffusion-weighted images. NMR in Biomedicine, 8:333-344, 1995. 441, 442, 443

2. P.J. Basser and C. Pierpaoli. Microstructural and physiological features of tissues elucidated by quantitative-diffusion-tensor MRI. J. Magn. Reson. Ser. B, 111:209219, 1996. 446

3. C. Beaulieu and P.S. Allen. Determinants of anisotropic water diffusion in nerves. Magn. Reson. Med., 31:394-400, 1994. 442

4. T. L. Chenevert, J. A Brunberg, and J. G. Pipe. Anisotropic diffusion in human white matter: Demonstration with MR techniques in vivo. Radiology, 177:401-405, 1990. 442

5. G. Gerig, O. Kübler, R. Kikinis, and F.A. Jolesz. Non-linear anisotropic filtering of MRI data. IEEE Transaction on Medical Imaging, 11(2):221-232, June 1992. 445

6. G. H. Granlund and H. Knutsson. Signal Processing for Computer Vision. Kluwer Academic Publishers, 1995. ISBN 0-7923-9530-1. 444

7. H. Gudbjartsson, S. E. Maier, R. V. Mulkern, I. Á. Mórocz, S. Patz, and F. A. Jolesz. Line scan diffusion imaging. Magn. Reson. Med., 36:509-519, 1996. 442, 443

8. M. E. Moseley, Y. Cohen, J. Kucharczyk, J. Mintorovitch, H. S. Asgari, M. F. Wendland, J. Tsuruda, and D. Norman. Diffusion-weighted MR imaging of anisotropic water diffusion in the central nervous system. Radiology, 176:439-445, 1990. 442

9. S. Peled, H. Gudbjartsson, C-F. Westin, R. Kikinis, and F.A. Jolesz. Magnetic Resonance Imaging shows Orientation and Asymmetry of White Matter Tracts. Brain Research, 780(1):27-33, January 1998. 450

10. C. Pierpaoli, P. Jezzard, P. J. Basser, A. Barnett, and G. Di Chiro. Diffusion tensor MR imaging of the human brain. Radiology, 201:637, 1996. 442

11. C. Poupon, J.-F. Mangin, F. Frouin, J. Régis, F. Poupon, M. Pachot-Clouard, D. Le Bihan, and I. Bloch. Regularization of mr diffusion tensor maps for tracking brain white matter bundles. In Proceedings of MICCAI'98, number ISSN 0302-9743 in Lecture Notes in Computer Science 1496. Springer Verlag, 1998. 445

12. R. Turner, D. le Bihan, J. Maier, R. Vavrek, L. K. Hedges, and J. Pekar. Echo planar imaging of intravoxel incoherent motions. Radiology, 177:407-414, 1990. 442

13. C-F. Westin and H. Knutsson. Extraction of local symmetries using tensor field filtering. In Proceedings of 2nd Singapore International Conference on Image Processing. IEEE Singapore Section, September 1992. 444

14. C-F. Westin and H. Knutsson. Estimation of Motion Vector Fields using Tensor Field Filtering. In Proceedings of IEEE International Conference on Image Processing, Austin, Texas, November 1994. IEEE. 444

15. C-F. Westin, S. Peled, H. Gudbjartsson, R.Kikinis, and F.A Jolesz. Geometrical diffusion measures for MRI from tensor basis analysis. In ISMRM '97, Vancouver, Canada, April 1997. 443

16. D. M. Wimberger, T. P. Roberts, A. J. Barkovich, L. M. Prayer, M. E. Moseley, and J. Kucharczyk. Identification of "premyelination" by diffusion-weighted MRI. J. Comp. Assist. Tomogr., 19(1):28-33, 1995. 442 